\title{
A VLC-based beacon location system for mobile applications
}

\author{
Guillermo del Campo-Jimenez, Jorge M. Perandones and F. J. Lopez-Hernandez
}

\begin{abstract}
This paper proposes a low cost and complexity indoor location and navigation system using visible light communications and a mobile device. LED lamps work as beacons transmitting an identifier code so a mobile device can know its location. Experimental designs for transmitter and receiver interfaces are presented and potential applications are discussed.
\end{abstract}

Keywords-indoor location; indoor navigation; beacon; visible light communications; LEDs

\section{INTRODUCTION}

Many nowadays mobile applications make use of location information to work or offer contextual services [1] [2]. Outdoors location information is obtained by the global positioning system (GPS) or the RF (mobile) signal. However, due to the signal attenuation caused by construction material, these methods do not work properly in indoor environments where a higher precision is required. During the last ten years different wireless techniques based on Wi-Fi [3], wireless sensor networks [4], Bluetooth [5], UWB [6], NFC [7] or IR [8] have been proposed. These techniques measure the object or person location according to received signal parameters and using specific algorithms. However, these location systems either require an additional node network infrastructure or have difficulties in acquiring accurate location due to multipath fading. Besides, the RF based systems are restricted in hospitals and other RF unfriendly areas.

There is an increasing trend in replacing fluorescent and halogen lamps by the more energy efficient SSL (Solid State Lamp). SSLs are based on visible LEDs which offer extended life cycles and higher conversion efficiencies compared to traditional incandescent lamps. The exponential rising on the number of SSL installed foresees a leadership of these devices in illumination.

VLC (Visible Light Communications) exploits the relative high switching speed of LEDs to use the light from SSL not only for lighting but also as a transmission channel. It offers a set of communication characteristics: low cost, medium to high speed, no electromagnetic interference, confined range, etc., which solves a number of RF (Radio frequency) technological issues: spectrum saturation, system interferences, security, etc. Due to these advantages, there has been a growing interest in research in VLC systems. Various modulation schemes have been proposed allowing transmission speed to reach hundreds of Mbps [9] for broadcast applications [10].

Regarding indoor localization, some VLC systems offer a promising alternative. Some VLC solutions have been proposed achieving accuracies of centimeters: recovering channel characteristics from incident light [11] or using carrier allocation modulation method [12] and calculating location with algorithms.

In this paper we propose an easy to implement and low cost VLC-based indoor location system. The system is formed by SSL lamps which act as beacons and a receiver interface connected to a mobile terminal where a visual application runs. The application takes advantage of the location information to offer high value services as indoor navigation or way finding through internet connection.

\section{SYSTEM DESCRIPTION}

\section{A. System overview}

The intended system consists on a mobile device (e.g. a mobile phone or a tablet) which receives location data from the lamp illuminating a determined area (see Fig. 1). Each lamp (or group of lamps) acts a beacon by sending repeatedly an identification code (ID) or coordinates. The mobile device receives these data and gets to know its exact position within the building.

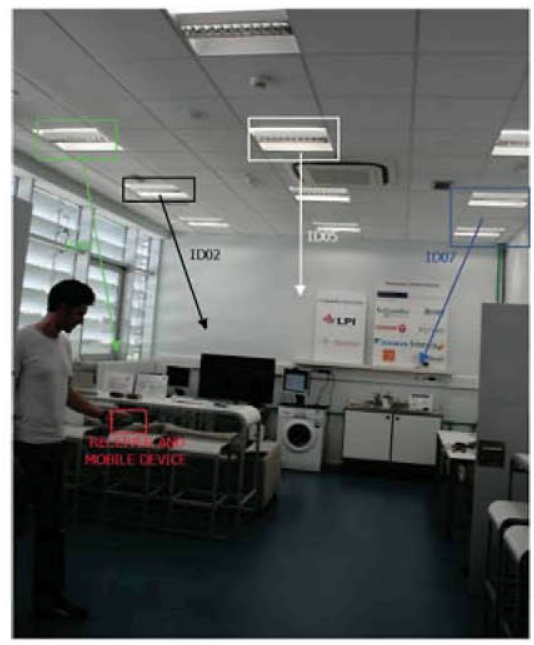

Figure 1. System overview 
Depending on the final application (e.g. navigation, way finding, object or personnel location) the system presents two different scenarios:

- Passive beacon: in this scenario the lamp continuously sends a previously assigned identifier. The mobile application makes use of this information to locate it and provide it with useful information. An example would be the gate finding in an airport. As the application knows exactly where the user is, it can guide him through the optimal itinerary. If internet connection is available, extra functionalities as gate change notifying and way recalculating can be addressed.

- Active beacon: in this scenario the lamp not only sends an identifier but also can provide extra information. An example would be highway tunnels where the lamps could send additional interesting information as traffic jams or accidents alerts.

\section{B. Coding scheme}

On-off keying (OOK) modulation is used for data transmission. In OOK modulation data are encoded by turning the LEDs on and off. The simplest way is to represent the symbol " 1 " with the light "on" state and the symbol " 0 " with the light "off" state (see Fig. 2). In order to maintain a constant average DC level, thus avoiding the illumination dependence on data, Manchester Coding [13] is employed. Dimming is achieved through the use of Pulse-width modulation (PWM) by changing the duty cycle of the pulses. Data rate is limited to 1-5 Mbps [14] by the switching times of LEDs, though is enough for location applications. When higher data rates are needed other modulations schemes as VPPM [15] or OFDM [16] can be used, though more complexity in both lamp driver and receiver is required.

\section{EXPERIMENTAL SETUP}

\section{A. Transmitter interface}

A simple and low cost microcontroller (TI's MSP430G2553) has been used for generating the OOKManchester encoded signal (Fig. 3).

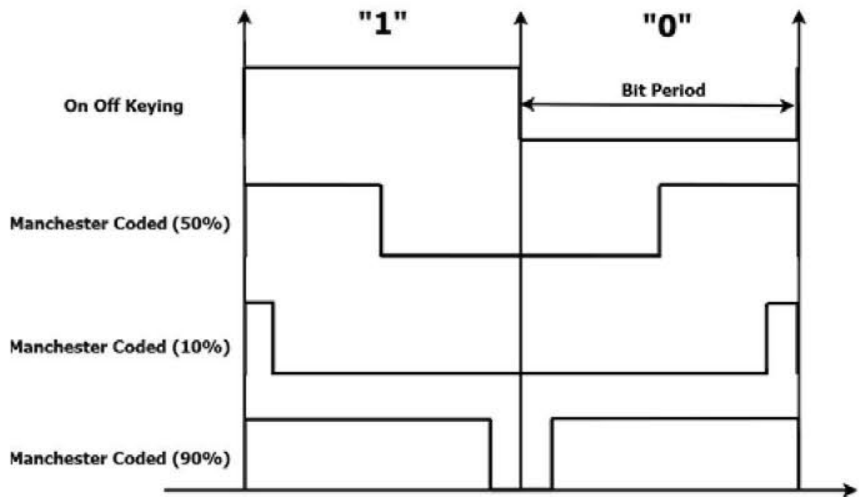

Figure 2. OOK modulation: simple, Manchester Coded (Dimming 50\%, 10\% and $90 \%$ )

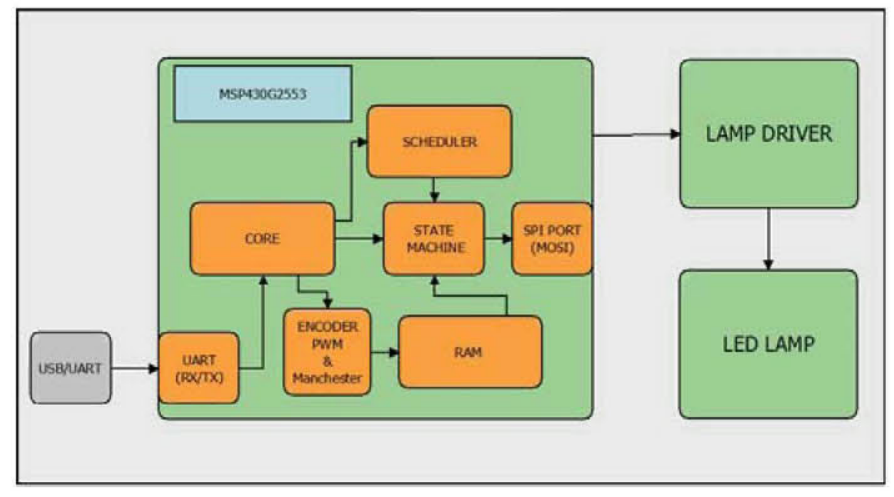

Figure 3. Transmitter interface block diagram

The use of the microcontroller allows defining communication parameters (message transmission period, duty cycle and message content) through an UART port. Personnel or object movement speed is relative low compared to transmission speed; then there is no need to send information continuously. However, lamp illumination role must be preserved. Hence, a state machine with three different working states has been programmed:

- IDLE: no data are being transmitted, though dimming functionality is enabled. Moderate switching frequency.

- DATA: data transmission state with no predefined length. Maximum switching frequency to allow highest transmission rate. ASCII coded.

- SYNC: it is a short state which transmits pulses of larger duration than in other states to signalize the beginning and ending of the DATA state.

Each state message is stored in the FLASH memory block until it replaced with a new one, optimizing the processing loads. The MOSI pin from the SPI port transmits the signal to the lamp driver. The SPI transmission buffer is filled by 16 digital pulses or chips. Light dimming (PWM) is achieved by the number of chips set to " 1 ", allowing illumination level to be changed from $6.25 \%$ to $93.75 \%$ in $6.25 \%$ steps (Fig. 4).

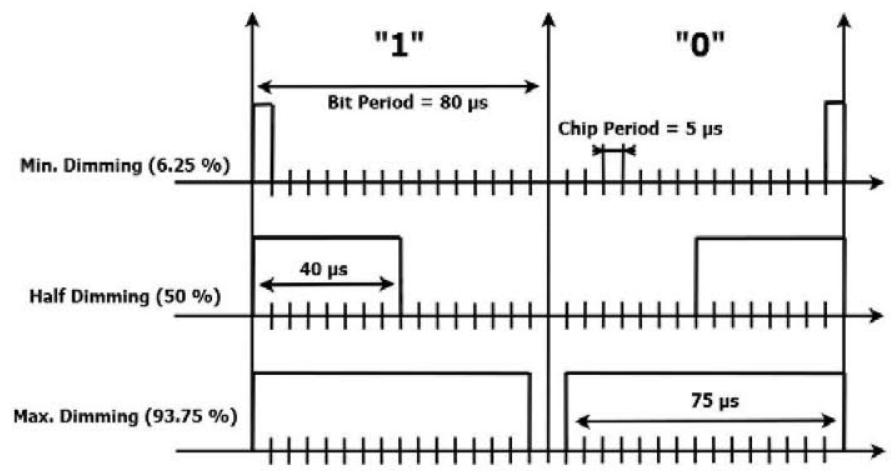

Figure 4. Chip and bit times for different illumination levels 


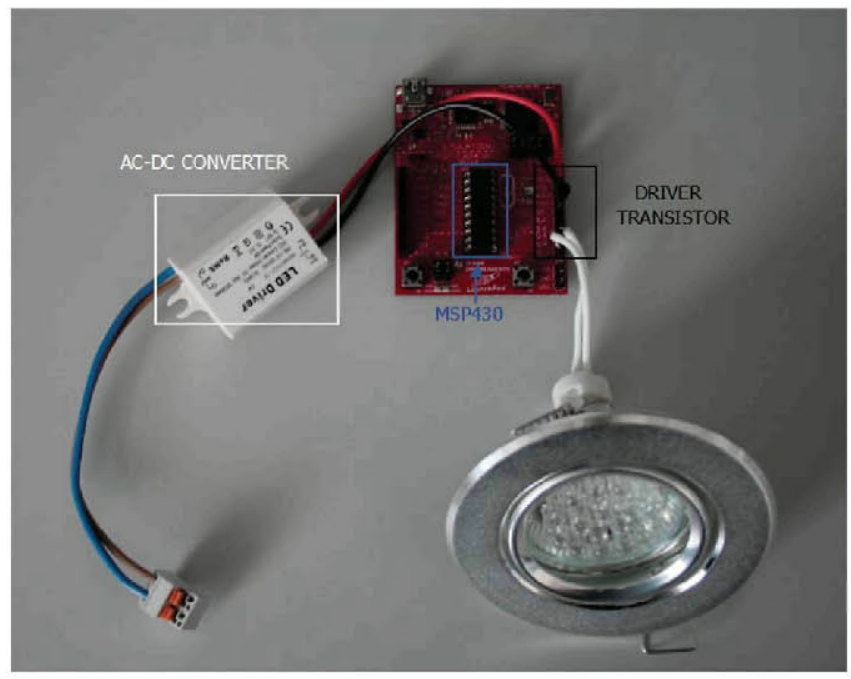

Figure 5. Experimental transmitter interface

As mentioned above, transmission speed can be limited by LED switching times. Maximum switching frequency of the installed LED lamps has been measured: $400 \mathrm{KHz}$. Hence the theoretical minimum pulse duration would be $2.5 \mu \mathrm{s}$. To minimize communication error, minimum pulse duration of 5 us $(200 \mathrm{KHz})$ has been chosen. As a data bit is formed by 16 chips, the minimum bit duration is 80 us (DATA state switching frequency is $12.5 \mathrm{KHz}$, while IDLE is $1.56 \mathrm{KHz}$ and SYNC is $776.7 \mathrm{~Hz}$ ).

A very simple lamp driver circuit is employed (Fig. 5). As electric line is used to power both the lamps and the microcontroller, voltage conversions are required. First, an $\mathrm{AC}$ DC converter adapts the mains $240 \mathrm{~V} \mathrm{AC}$ to $12 \mathrm{~V} \mathrm{DC}$, which is the lamp voltage. An additional DC-DC converter is used to power the MSP430 (5V). As the SPI output from the MSP430 does not provide enough current to switch the lamp, a MOSFET transistor is used. Due to the switching regime, the transistor dissipates low electrical power, making a heat sink unnecessary. Also, the MOS case is very small reducing the size of the system.

\section{B. Receiver interface}

The receiver interface is formed by a transimpedance amplifier, followed by a demodulating and data recovering system (Fig. 6). The illumination signal is detected and converted to a current signal by a photodiode. The transimpedance amplifier transforms the current signal to an analog voltage signal, followed by a comparator which eliminates noise and prepares the signal for the demodulation process where another MSP430 is employed.

The MSP430 uses the GPIO port to detect the pulse edge and triggering an interruption. Time between interruptions is measured and the pulse duration calculated. Similarly to the transmitter interface, the demodulation process is based in a state machine. If no SYNC signal is received the system assumes that it is in the IDLE state and calculates de duty cycle. When a SYNC signal is detected, the system demodulates the data and stores it until the message is complete (another SYNC signal is detected).

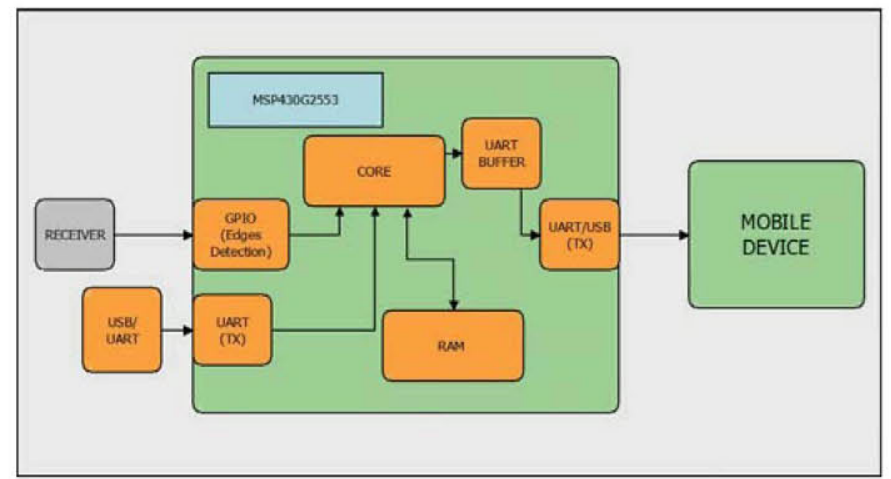

Figure 6. Receiver interface block diagram

Finally, it sends the message to the mobile device through the USB port.

Before installing the system, different laboratory communications tests have been conducted using a 1 W LED spotlight bulb. Error free communication is achieved for up to a $4.5 \mathrm{~m}$ range and varying the orientation \pm 30 degrees. Besides, changing illumination level does not affect the transmission throughput, i.e. it works with illumination almost zero. These results prove that the system is suitable for larger buildings when using luminaries with higher power.

\section{APPLICATIONS}

As a preliminary example for testing the system, an indoor navigation application has been designed. The basic application consists of a map which shows the user current location, by managing a correspondence table between IDs and map areas. Besides, the user can set the desired destination on the map and the application calculates the optimal route to reach his/her destination based on the current location. If the mobile device is equipped with camera, it offers augmented reality functionalities: it can show additional information about recognized elements.

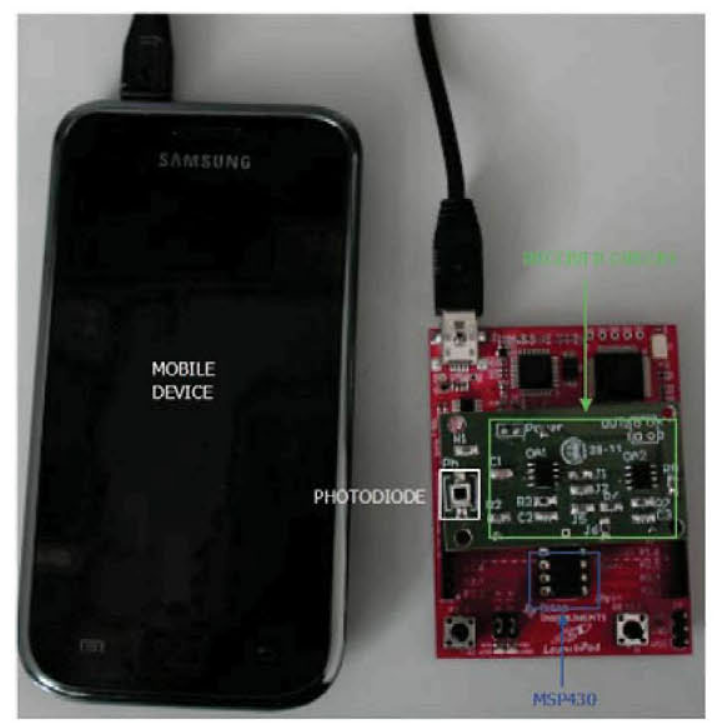

Figure 7. Experimental receiver interface 


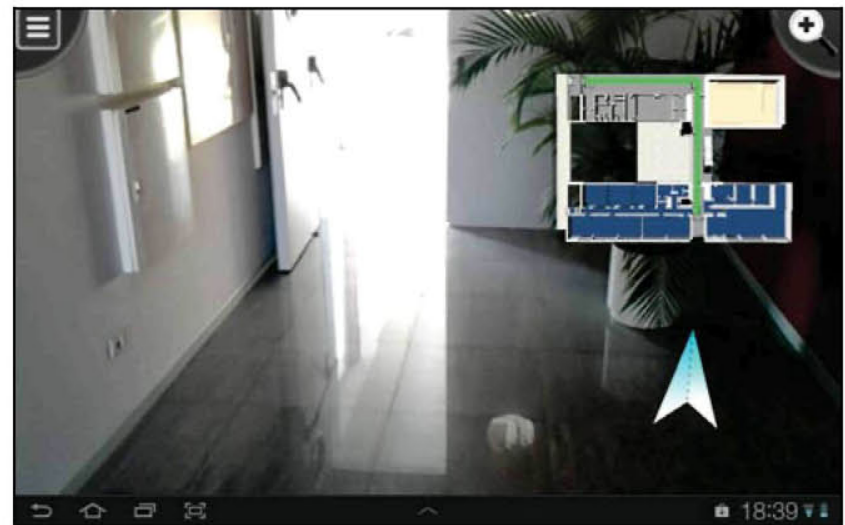

Figure 8. Navigation application graphic interface

This guiding capability is especially well suited to public buildings, being hospitals and airports the most significant. In both cases, the user can be easily guided from his/her entrance to his/her destination. If the receiver is a mobile phone, the building information system can be aware of the presence and position of the user, being able of offer him/her useful information. In addition, using tunnel illumination sources and road traffic lights as active beacons could help drivers by providing them with real time knowledge about nearby events as road construction or traffic jams and helping them to choose the best route. Besides, contextual information as closest petrol station or services can be supplied.

\section{CONCLUSIONS}

With the future increase use of LEDs as illumination sources, VLC appears as an alternative to traditional RF communications, being indoor location and navigation one of its potential applications.

This paper presents the preliminary design and implementations of an indoor location system using LED lamps as beacons through VLC communications. Design goal was to optimize cost and installation complexity. Laboratory tests and a first implementation show the usability of the system and further adaptation to bigger building as shopping malls. Two different application scenarios have proposed: a passive beacon continuously sending location identifier, intended for way finding in airports or hospitals; and an active beacon sending additional information.

\section{REFERENCES}

[1] Coronel, N.O.M.; Ortiz Hernandez, J.; Saenz Sanchez, S.; Serna, J.G.G., "Integration of Usability Into the Development of Mobile Computing Environments Applied to Contextual Location Based Services (LBS)," Electronics, Robotics and Automotive Mechanics Conference (CERMA), 2011 IEEE, vol., no., pp.409,414, 15-18 Nov. 2011
[2] Chon, Y.; Talipov, E.; Shin, H.; Cha, H., "SmartDC: Mobility Prediction-based Adaptive Duty Cycling for Everyday Location Monitoring," Mobile Computing, IEEE Transactions on , vol.PP, no.99, pp. $1,1,0$

[3] Bisio, I.; Pan, R.L.C.; Lavagetto, F.; Marchese, M.; Sciarrone, A.; Fra, C.; Valla, M., "Smartphone-based automatic place recognition with WiFi signals for location-aware services," Communications (ICC), 2012 IEEE International Conference on , vol., no., pp.4943,4948, 10-15 June 2012

[4] Zhou Hui; Li Dan-Mei; Shao Shi-Huang; Xu Chen, "A Novel Intelligent Estimation Algorithm in WSN Location Based On Free Search," Wireless Communications, Networking and Mobile Computing, 2007. WiCom 2007. International Conference on, vol., no., pp.2629,2632, 2125 Sept. 2007

[5] Gonzalez-Castano, F.J.; Garcia-Reinoso, J., "Bluetooth location networks," Global Telecommunications Conference, 2002. GLOBECOM '02. IEEE, vol.1, no., pp.233,237 vol.1, 17-21 Nov. 2002

[6] Correal, N.S.; Kyperountas, S.; Shi, Q.; Welborn, M., "An UWB relative location system," Ultra Wideband Systems and Technologies, 2003 IEEE Conference on, vol., no., pp.394,397, 16-19 Nov. 2003

[7] Siira, E.; Tuikka, T.; Tormanen, V., "Location-Based Mobile Wiki Using NFC Tag Infrastructure," Near Field Communication, 2009. NFC '09. First International Workshop on, vol., no., pp.56,60, 24-24 Feb. 2009

[8] McGillem, C.D.; Rappaport, T.S., "Infra-red location system for navigation of autonomous vehicles," Robotics and Automation, 1988. Proceedings., 1988 IEEE International Conference on , vol., no., pp.1236,1238 vol.2, 24-29 Apr 1988

[9] Vucic, J.; Kottke, C.; Nerreter, S.; Langer, K.-D.; Walewski, J.W., "513 Mbit/s Visible Light Communications Link Based on DMT-Modulation of a White LED," Lightwave Technology, Journal of , vol.28, no.24, pp.3512,3518, Dec. 15,2010

[10] Poves, E.; Del Campo-Jimenez, G.; Lopez-Hernandez, F. J., "VLCbased light-weight portable user interface for in-house applications," Consumer Communications and Networking Conference (CCNC), 2011 IEEE , vol., no., pp.365,367, 9-12 Jan. 2011

[11] Zhou Zhou; Mohsen Kavehrad; Peng Deng, "Indoor positioning algorithm using light-emitting diode visible light communications," Optical Engineering 51 (8) SPIE, August 2012

[12] Hyun-Seung Kim; Deok-Rae Kim; Se-Hoon Yang; Yong-Hwan Son; Sang-Kook Han, "Indoor positioning system based on carrier allocation visible light communication," Quantum Electronics Conference \& Lasers and Electro-Optics (CLEO/IQEC/PACIFIC RIM), 2011 , vol., no., pp.787,789, Aug. 28 2011-Sept. 12011

[13] IEEE Standard for Local and Metropolitan Area Networks--Part 15.7: Short-Range Wireless Optical Communication Using Visible Light," IEEE Std 802.15.7-2011, vol., no., pp.1,309, Sept. 62011

[14] Little, T. D C; Dib, P.; Shah, K.; Barraford, N.; Gallagher, B., "Using LED Lighting for Ubiquitous Indoor Wireless Networking," Networking and Communications, 2008. WIMOB '08. IEEE International Conference on Wireless and Mobile Computing, , vol., no., pp.373,378, 12-14 Oct. 2008

[15] Lopez-Hernandez, F. J.; Campo-Jimenez, G.; Poves, E.; MartinGonzalez, J. A., "LED jitter-induced limitation effects in the baud rate of VLC systems," Consumer Electronics (ICCE), 2011 IEEE International Conference on, vol., no., pp.39,40, 9-12 Jan. 2011

[16] Hashemi, S. K.; Ghassemlooy, Z.; Chao, L.; Benhaddou, D., "Orthogonal frequency division multiplexing for indoor optical wireless communications using visible light LEDs," Communication Systems, Networks and Digital Signal Processing, 2008. CNSDSP 2008. 6th International Symposium on, vol., no., pp.174,178, 25-25 July 2008 